\title{
Universidad y empresa ante la doble crisis del periodismo tradicional. Propuestas y reflexiones sobre la modificación sustancial del escenario periodístico. El clúster periodístico
}

\author{
Fernando Sabés Turmo \\ Universitat Autònoma de Barcelona \\ José Juan Verón Lassa \\ Universidad San Jorge de Zaragoza
}

\section{Palabras clave}

Periodismo, crisis, estudios de comunicación, emprendedor y clusters.

\section{Resumen}

Este artículo analiza los cambios que está viviendo la profesión periodística partiendo de dos variables: la tecnología y la actual crisis que afecta directamente al sector. En el texto se aborda la necesidad de profundizar en las raíces del periodismo, en las formas de hacer, y no caer únicamente en el perfil tecnológico que en los últimos años ha invadido la profesión. Aborda la necesidad de debatir sobre los títulos en las facultades de la comunicación acercándose a la realidad cambiante del mundo profesional y aboga por la emprendeduría y la creación de clusters periodísticos como vías de futuro. 
University and business facing the double crisis of traditional journalism. Proposals and reflections on the substantial change in the journalistic scene. The cluster reporting

\title{
Key words
}

Journalism, crisis, communication studies, entrepreneurship, clusters.

\begin{abstract}
This paper analyzes the changes that the journalistic profession is experiencing from two variables: technology and the current crisis that directly affects the sector. The text talks about the need to deepen the roots of journalism, in the ways of doing, and avoids the technological profile that has invaded the profession in recent years. It raises the need to discuss the titles in Faculties of communication getting closer to the changing reality of professionals and it defends entrepreneurship and the creation of journalistic clusters as means of future development.
\end{abstract}

\section{Autores}

Fernando Sabés Turmo [fernando.sabes@uab.cat]es profesor del departamento de Periodismo y Ciencias de la Comunicación de la Universitat Autònoma de Barcelona.

José Juan Verón Lassa [jjveron@usj.es] es profesor de la Facultad de Comunicación de la Universidad San Jorge de Zaragoza.

\section{Créditos}

El Proyecto TICLaude, Bolsa de Jóvenes Emprendedores en TIC, se desarrolla por la Facultat de Ciències de la Comunicació de la Universitat Autònoma de Barcelona y está subvencionado por el Ministerio de Educación. Para más información se puede consultar http://www.ticlaude.info 


\title{
1. Introducción
}

La actual situación de la profesión periodística no emite señales de optimismo hacia su futuro. La recesión económica ha caído como una losa en un sector que ya sufría una profunda crisis propia de modelo de negocio, derivada de la irrupción de las tecnologías de la información. Sin embargo, la carrera continúa siendo una de las preferidas por los jóvenes universitarios españoles, que siguen escogiendo de forma mayoritaria los estudios vinculados a la comunicación.

Según el Observatorio de Crisis de la Federación de Asociación de Periodistas de España ${ }^{1}$, desde noviembre de 2008 y hasta el mes de mayo de 2012 se han destruido un total de 6.234 puestos de trabajo y han cerrado 57 medios de comunicación .

Por otro lado, la valoración pública que realiza la sociedad de la profesión es negativa, según los datos que periódicamente publica, por ejemplo, en sus barómetros el Centro de Investigaciones Sociológicas ${ }^{2}$.

\begin{abstract}
«En el plano social, la demanda de estudios de periodismo parece ir en contra de las conclusiones que podrían extraerse de un análisis racional de oportunidades que considere la evolución del mercado de trabajo comparado con las inversiones necesarias, fundamentalmente económicas y de tiempo vital; se evidencia una sobrepresión social para acceder a los estudios de comunicación en toda la universidad española a pesar de las dificultades que tiene el sistema de medios para proporcionar ocupación a los egresados. En contraposición, puede argumentarse que los periodistas formados adecuadamente estarán capacitados para desarrollar múltiples ocupaciones, no sólo redaccionales, y podrán trabajar en sistemas de medios de otros países» (Pestano, Rodríguez y Del Ponti, 2011:411).
\end{abstract}

Existen numerosos estudios que abordan las razones que llevan a los estudiantes de comunicación a escoger su titulación. «El factor de la motivación externa, según expresan los futuros estudiantes de comunicación, es el que menos les influye a la hora de decidir los grados que quieren realizar. Su decisión no tiene que ver con moda alguna, ni está en relación con el grado de dificultad de los estudios y, no es consecuencia de los consejos de sus padres y amigos. Los jóvenes que van a comenzar estos estudios superiores se mueven con mucha mayor intensidad por otro tipo de motivaciones más personales» (Peinado y Fernández, 2011: 395).

Esta situación conduce a reflexionar sobre el futuro del periodismo tal y como se ha entendido hasta ahora, un aspecto que evidentemente debe llevar acompañada una profunda reflexión sobre los estudios que se imparten en las facultades de comunicación, de acuerdo a la evolución cada vez más rápida del sector, que tiene que adaptarse a unos nuevos tiempos de forma inmediata.

Algunos de estos aspectos se abordan en este trabajo en el que se analizan las modificaciones que se están produciendo en el sector por la transformación digital, el papel de las universidades como formadoras de comunicadores, así como

\footnotetext{
$1 \mathrm{http}: / /$ www.fape.es

2 http://www.cis.es
} 
un cambio de modelo de empleador y empleado en la materia, apostando, entre otros aspectos, por la emprendeduría, la innovación y la incorporación del periodista a ámbitos que hasta el momento habían sido ignorados por la profesión.

\section{Cambios por la invasión digital}

«No es necesario ser profeta para aventurar que la muerte de los diarios impresos en papel no demorará más allá del año 2020». Así de contundente se expresaba en 1997 Martínez Albertos (1997:24) y los datos de los últimos años en cuanto a difusión, inserción publicitaria y cierre de medios, como se verá con detalle más adelante, apuntan completamente en esa dirección.

Las tecnologías de la información y de la comunicación, calificadas como nuevas hace unos años y que en la actualidad son las que todos los periodistas y la mayor parte de ciudadanos utilizan en su día a día, están condicionando por completo la forma como se genera información y como se consume, cambiando, en parte, el tradicional paradigma de la comunicación (emisor, receptor, mensaje, canal y feedback).

Estas tecnologías ya han dejado de ser únicamente soportes en los que poder publicar una cantidad ingente de información y de opinión. Han ido cambiando el modo y la manera como circula el contenido, desde su generación hasta el consumo y, sobre todo, un aspecto que se ha potenciado sustancialmente, la retroalimentación. En esta línea, el público ha dejado su tradicional papel pasivo para, al menos en teoría y cada vez más en la práctica, ser parte muy activa del proceso comunicativo.

Es lo que Salaverría y Negredo (2008: 21) llaman la tormenta perfecta:

«En este nuevo contexto de mercado, dificultado además por un entorno económico recesivo, los medios tradicionales se enfrentan a lo que podría describirse como la tormenta perfecta: aumento de los costes de producción, estancamiento o caída de la difusión, menor inversión publicitaria, desaparición de puntos de venta y aumento de la competencia. Todo al mismo tiempo».

Estos cambios llevan a reflexionar sobre la redefinición misma de la profesión periodística. Este replanteamiento debe comenzar en las facultades de ciencias de la comunicación. Sin embargo, en demasiadas ocasiones parecen, incluso en los nuevos planes de estudios adaptados a los grados, ancladas a la tradicional división por soportes.

Cuando se aborda la docencia en las facultades de comunicación, hasta el momento se ha tendido a plantear Internet (si bien, hace tiempo que los nuevos modos y soportes han superado en mucho el propio concepto de Internet y se han acuñado otros términos como Web 2.0) como un medio más. De igual manera sucede cuando se plantea el desarrollo de la profesión periodística. 
Por otro lado, se encuentra muy próxima la percepción de que en breve Internet, con todas sus evoluciones y variantes, debe ser el más importante de todos los medios, en sustitución de la actual primacía de la televisión, habiendo enterrado en más de una ocasión a los medios tradicionales, esencialmente a la prensa en papel. Se trata de un fenómeno que ya se está dando en las generaciones más jóvenes, que dedican más tiempo a Internet y las redes sociales que al consumo de televisión.

Según la encuesta Social Media Tracker de 2012 (sexta oleada del estudio), los españoles pasan una media de 9 horas a la semana frente al televisor, dedican 5 horas a la radio, 3 horas a la lectura de revistas, 4 horas a la lectura de diarios, 13 horas a Internet y 5 horas a las redes sociales (tiempo que se amplía hasta las 9 horas para los menores de 24 años) $)^{3}$.

En esta línea, hace unos años, los futurólogos de la comunicación avanzaban que la prensa en papel de pago podía desaparecer ante el auge de la prensa gratuita. Aunque no sucedió dicho vaticinio, sí que se observó un movimiento en los anunciantes que sacudió el modelo tradicional de negocio.

La crisis económica ha acentuado un retroceso muy importante en la inversión publicitaria que afecta a la prensa y, particularmente, ha hundido buena parte de las cabeceras gratuitas que habían proliferado sobre todo en las grandes ciudades. De hecho, existe una larga nómina que incluía cabeceras como Metro y $A D N$, además de algunas iniciativas; casi todas ellas desaparecidas. La difusión de los diarios gratuitos nacionales había caído de 4,9 millones en 2006 a 1,5 millones a final de 2011 y en mayo de 2012 seguía bajando, con 20 Minutos por debajo de los 700.000 ejemplares y Qué! rondando los 600.000, según datos de Newspaper Innovation ${ }^{4}$. Otro dato ilustrativo: el número de ediciones locales en este tiempo se había reducido de 35 a 14 .

En cualquier caso, pese a los cambios cada vez más rápidos que afectan a los medios de comunicación, la televisión es todavía el medio al que se le destina mayor inversión publicitaria y que arrastra más consumidores, sin olvidar que es una referencia mediática (prestigio, que se entiende casi siempre como sinónimo de espectáculo).

Según el Estudio de inversión publicitaria en medios digitales en España en 2011 realizado por el Interactive Advertising Bureau ${ }^{5}$ (IAB) y publicado en marzo de 2012, en el último año, solo dos medios o soportes han crecido en facturación: los digitales $(12,6 \%)$ y el cine $(5,8 \%)$. Aunque es cierto que la inversión publicitaria en medios digitales está muy lejos de la que se realiza en televisión (ver Tabla 1), comienza ya a acercarse a la que se realiza en los diarios y ha superado a medios tradicionales como la radio, las vallas publicitarias.

3 http://www.slideshare.net/UMSpain/wave6-espaol

4 http://www.newspaperinnovation.com/index.php/2012/05/22/spainish-free-circulation-drops/

5 www.iabspain.net 
Estas cifras son especialmente significativas en un contexto en el que el conjunto del dinero destinado a publicidad ha descendido un $6 \%$ entre 2010 y 2011 . También es muy importante el hecho de que el porcentaje de inversión publicitaria en medios digitales no ha dejado de crecer desde 2005 (IAB, 2012), mientras que en televisión y, especialmente, en diarios y revistas se observa una marcada tendencia a la baja en los últimos 7 años. Por tanto, teniendo en cuenta que la crisis económica se manifestó a partir de finales de 2008, sería engañoso achacarle el declive en la rentabilidad de estos medios.

Tabla 1. Evolución de la inversión publicitaria en España entre 2010 y 2011 (miles de euros)

\begin{tabular}{|l|c|c|c|}
\hline & $\mathbf{2 0 1 0}$ & $\mathbf{2 0 1 1}$ & Incremento (\%) \\
\hline Televisión & $2.471,90$ & $2.237,20$ & $-9,5$ \\
\hline Diarios & $1.124,40$ & 967 & -14 \\
\hline Digitales & 798,9 & 899,2 & 12,6 \\
\hline Radio & 548,5 & 524,9 & $-4,3$ \\
\hline Exterior & 420,8 & 402,8 & $-4,3$ \\
\hline Revistas & 397,8 & 381,1 & $-4,2$ \\
\hline Dominicales & 72,2 & 67,1 & -7 \\
\hline Cine & 24,4 & 25,8 & 5,8 \\
\hline Total & $5.858,8$ & $5.505,1$ & -6 \\
\hline
\end{tabular}

Fuentes: IAB e Infoadex

La realidad está cambiando con más rapidez de lo que muchos esperaban. La irrupción masiva de las tecnologías de la red en los hogares, en los lugares de trabajo y también en los de ocio hace que esta tendencia se esté acelerando, aunque hay que reconocer que existe todavía una brecha digital entre los públicos de mayor edad, con un impacto cada vez más reducido.

Entre los jóvenes, Internet es el soporte preferido, esencialmente como entretenimiento. Una reflexión que excede este informe es la naturaleza de la información y la cantidad que consumen los jóvenes en la actualidad. Todo ello se ha acelerado con la generalización de Internet móvil.

«A diferencia de lo que ocurre en el medio impreso, los cambios en Internet se producen a gran velocidad, lo que ha provocado una evolución vertiginosa de los modelos de prensa. Pero el proceso no ha hecho sino comenzar» (Amaral y Gimeno, 2009: 15).

Internet se ha consolidado como un modo de transmisión de todo tipo de contenido y, especialmente en el último lustro, su uso como soporte informativo va en imparable crecimiento. Es cierto que todavía puede resultar limitado el im- 
pacto en nuestra sociedad de las informaciones que son publicadas en la red si no aparecen también en los medios tradicionales; pero todo apunta, en función de los cambios de hábitos descritos en cuanto al consumo de medios, que esta dinámica se encuentra un acelerado proceso de cambio.

De hecho, en los últimos meses se están produciendo fenómenos en el sentido contrario, con informaciones que alcanzan gran virulencia en redes sociales y, solo posteriormente, son tratadas en los medios convencionales, que informan también de sus resultados. Baste recordar la campaña de abril de 2011 \# eurodiputadoscaraduras sobre el uso de la primera clase por parte de los eurodiputa$\operatorname{dos}^{6}$ o la campaña que tuvo lugar entre mayo y junio de 2012 \#vaportinacho, a raíz de la que Bruce Springsteen ${ }^{7}$ dedicó una canción en un concierto a un joven seguidor que murió sin haber podido acudir a uno de sus conciertos.

Un estudio realizado por LinkedIn indica que la prensa escrita es el sector económico con una mayor caída en términos relativos $(-28,4 \%)$ en el periodo 2007-2011 en Estados Unidos. El mismo informe, publicado a comienzos de 2012 señala que Internet y las publicaciones online, con un 24,6\% y 24,3\% respectivamente, son dos de los sectores con un mayor crecimiento en el mismo periodo $^{8}$.

Los datos apuntan claramente una tendencia de cambio y hacia la universalización de las tecnologías digitales. Incluso la televisión ha dado el salto a las nuevas tecnologías y desde el 3 de abril de 2010, el conjunto de España cuenta con cobertura de Televisión Digital Terrestre (TDT). Según los datos del Ministerio de Industria, el 98\% del territorio nacional está cubierto por esta tecnología. Además, se procedió a un apagón analógico, que dejó sin uso la tecnología tradicional. De este modo, la TDT se han convertido en la primera tecnología digital de extensión y uso universal en el ámbito español.

Los últimos datos disponibles indican que el 97,4 \% de las empresas cuenta con conexión a Internet (INE, 2011) y prácticamente todas $(99,4 \%)$ poseen conexión de banda ancha. Esta cifra es algo más baja en los hogares y se sitúa en el $61,9 \%$, aunque las empresas marcan claramente el camino. De hecho, en los últimos años, el número de hogares con Internet se está incrementando en más de un $2 \%$ anual respecto del número total de hogares.

También es destacable cómo el acceso a Internet en los hogares es mayor en el entorno rural. Esta circunstancia hace que la temida brecha digital esté quedando en un tópico ampliamente superado por la mayor parte de los ciudadanos.

Las tendencias en el consumo se están modificando a alta velocidad. Los smartphones y las tabletas han acelerado un cambio que se inició con los ordenadores y se impulsó con la generalización de los portátiles. La forma en la que

6 http://sociedad.elpais.com/sociedad/2011/04/06/actualidad/1302040817_850215.html

7 http:/www.elmundo.es/elmundo/2012/06/18/cultura/1339970473.html

8 http://blog.linkedin.com/2012/03/08/economic-report/ 
los ciudadanos reciben, leen y comparten la información nada tiene que ver con la que era habitual en la sociedad de hace simplemente un lustro. Además, esa portabilidad permite también estudiar nuevos sistemas de negocio y valorar las posibilidades que ofrecen para rentabilizar económicamente este tipo de comunicación, un aspecto vital para el futuro del periodismo.

Es una cuestión que está en debate desde hace años tanto en las redacciones como en las facultades de comunicación. Así, algunos medios se han lanzado a desarrollar laboratorios de experimentación, que hasta la fecha han tenido poco éxito. Mientras, la Universidad ha reflexionado por su cuenta, intentando aportar algo de luz dentro de un túnel complejo del que la industria periodística todavía no ha encontrado cómo salir.

En este sentido cabe citar aportaciones interesantes como la realizada por Flores y Aguado (2005), que analizaron los modelos aplicados hasta la fecha por distintas empresas. Lo más interesante de esta publicación es observar cómo 7 años después, el debate ha variado muy poco (gratuidad o pago, comercio electrónico, publicidad online), pese a haber entrado de lleno en una evolución desde el simple Internet a la Web 2.0. En cualquier caso, aportaciones de este tipo tienen la virtud de mostrar como el tablero de juego se ha modificado, dejando casi inservibles las estructuras productivas y de negocio sobre las que se desarrollaba la profesión periodística.

Y si la tendencia en la producción, distribución y consumo se modifica, parece lógico que debiera variarse la formación que se está dando a los nuevos comunicadores, esencialmente periodistas. Los profesionales que tendrán que habituarse a un soporte en constante evolución y que, además de sus características propias, ya ha logrado una integración de los soportes tradicionales: prensa, radio y televisión.

Todos estos datos ponen de manifiesto que el futuro del periodismo no tiene que ceñirse en el debate entre tecnólogos y no tecnólogos, sino que ha de ser mucho más profundo y debe plantearse en como la elaboración y la distribución de la información debe cambiar, como ya lo hizo también en el pasado con la aparición de nuevos medios como fueron la radio y la televisión. Pero, además, también habrá que analizar el modo en el que varían las formas de consumo y de retroalimentación; es decir, de participación activa del público que en muchas ocasiones deja de jugar ese rol y se convierte en productor. De hecho, se está acuñando un nuevo término - prosumidor-, que viene a definir este fenómeno. Y, lo que hoy resulta especialmente importante y urgente, cuál será el nuevo modelo (o modelos) de negocio que permitirá que el periodismo siga siendo una profesión con la que miles de personas puedan ganarse la vida dignamente.

En cualquier caso, hay que partir de una premisa fundamental: es imposible hacer periodismo sin periodistas. «La ruptura de paradigmas y el impacto de las transformaciones no deben impedirnos observar lo que pervive del periodismo heredado del pasado siglo. Si analizamos la preceptiva periodística que preside 
la práctica profesional y los códigos deontológicos, constatamos que los principales valores éticos del periodismo y que defendieron los periodistas durante el pasado siglo permanecen en la actualidad» (López, 2011).

«La obsolescencia de productos, estrategias, métodos y herramientas no implica que la misión del periodismo haya perdido sentido, incluso ante la enormidad de los volúmenes de información que la audiencia consume y manipula a diario. Lidiar con ello es ahora, también, nuestro trabajo. Entender cómo infiltrarse en ese problema es ahora, también, nuestra obligación, pero sobre todo una oportunidad invalorable» (Mancini, 2011: 119-120).

El periodismo va mucho más allá de ofrecer información sobre algún hecho o acontecimiento; el periodismo es una forma de trabajar la información, con unas fuentes coherentes y bien abordadas, con los datos que se ofrecen que sean solventes y contrastados... Es decir, trabajar la información con criterio.

\section{Universidad y mundo profesional}

Los estudios de Periodismo, y también los de Comunicación Audiovisual y Publicidad y Relaciones Públicas, han tenido un extraordinario auge en los últimos años, tanto por la implantación de un número importante de centros que los imparten (públicos y privados) como por la cantidad de alumnos que solicitan estas titulaciones como primera opción.

\footnotetext{
«El cambio tecnológico y social se refleja claramente en el periódico como medio. En todas las décadas del siglo XIX, época de apogeo del periódico, surgían nuevos estratos en la esfera periodística, desde deportes o asuntos delictivos hasta categorías de hogar y de ocio y añadían nuevos segmentos al lector y a los anunciantes. Desde 1945, el mundo ha presenciado la disolución de antiguas clases, partidos e ideologías mientras que el periódico que las expresaba encontraba su sitio en nuevos mercados de masas. En nuestra era, el cambio tecnológico está revelando transformaciones masivas en las formas en que las personas se autodefinen y los medios de comunicación deben cubrir las necesidades de todas las personas en sociedades con múltiples identidades. Ahí reside la angustia del periódico, que quedará enterrado al lado de su generación actual de lectores de avanzada edad, o puede que dé un salto espectacular hacia un nuevo medio que aguarda» (Smith, 2011)
}

Esta situación se produce en un momento en el que la incorporación laboral de estos jóvenes al mercado tradicional de los medios de comunicación es más que complicada ante la crisis económica global y que, como se ha dicho, está acentuada con la crisis profunda de los propios medios tradicionales y la falta de un nuevo modelo de negocio para los medios presentes en las nuevas plataformas.

Es pues un momento de cambios globales en la comunicación, desde la producción a la distribución y al consumo. Por tanto, estas variaciones también han de afectar de forma directa a la preparación que reciben estos jóvenes que quieren ser periodistas y que han visto como en pocos años el mercado de la comunicación se ha trasformado.

Paralelamente, la situación de crisis económica ha llevado a una precariedad laboral que hace temblar los cimientos de la profesión y la valoración de la misma por parte de la ciudadanía está cayendo constantemente. 
Es decir, que los medios de comunicación tienen ante sí una serie de cambios profundos, que hacen tambalear los principios sobre los que se habían asentado hasta el momento. «En un intento por ajustarse a la nueva sociedad, mudan sus procesos de producción, sus herramientas e infraestructuras, sus códigos comunicativos y, como se ve, incluso hasta sus modelos de negocio tradicionales» (Salaverría y Negredo 2008: 31).

En este importante momento, la Universidad y el mundo profesional deberían realizar un esfuerzo por aproximarse. La percepción de distanciamiento entre ambas esferas es notable y no se ha reducido en absoluto, o al menos esa es la sensación que se percibe sobre todo en el ámbito profesional. Y ello, a pesar de que esta dinámica debería haberse roto con la implantación total de los planes adaptados al Espacio Europeo de Educación Superior y que fueron concebidos, al menos en parte, para ello, aunque evidentemente la preparación académica debe superar el estadio de ser una mera formación profesional.

La situación no es sencilla. Además de que la crisis económica ha provocado el cierre de medios de comunicación, la reducción de las plantillas y la precarización de las mismas, entre otras consecuencias, no se puede perder de vista que la comunicación evoluciona a pasos agigantados y los tradicionales medios de comunicación no parece que vayan a ser los nichos de mercado en los que se van a incorporar los nuevos graduados en periodismo, sino que deberán ser los nuevos medios con Internet como soporte y sobre todo el autoempleo, dos de los ámbitos en los que más deberán incidir estos jóvenes que buscan hacerse un hueco en el sector.

Todo ello, con un condicionante claro y es que el sistema de producción de información ha cambiado, también la distribución y el consumo, incorporándose un auténtico feedback que en épocas pasadas era más una ilusión que una realidad pero que ahora es uno de los ejes esenciales en los que se sustenta la comunicación de masas.

En este sentido, cobran interés reflexiones como las realizadas por Xosé López (2010) en su libro La metamorfosis del periodismo, en donde aborda al mismo tiempo la adaptación de las estructuras académica y profesional ante la irrupción del ciberperiodismo. López incluye algunas recomendaciones y experiencias interesantes, como la necesidad de que los periodistas realicen de manera sistemática un proceso de formación continua en sus empresas, algo que hasta la fecha muy pocas de ellas han favorecido.

Una reflexión similar realiza Mancini, que concluye: «No tengo la menor idea acerca de cómo debe ser diseñada la formación de los nuevos periodistas, porque no tengo la menor idea de qué tenemos que producir» (2011: 101).

Los autores citados coinciden en que los principios del periodismo se mantienen independientemente de las novedades tecnológicas e incluso sociales, un aspecto clave en la formación de nuevos profesionales. Es más, dichos principios clá- 
sicos tienden a reafirmarse y a profundizar en su importancia por el fenómeno de la sobreabundancia de la información.

\begin{abstract}
«El público va a seguir demandando información fiable, y solo el periodista, por los estándares con los que trabaja, va a ser capaz de ofrecer garantías de ello. Al final, la credibilidad es el último reducto del periodismo, su última trinchera. Que el periodismo sea o no sea en el siglo XXI va a ser una cuestión de confianza, una cuestión de que el trabajo periodístico continúe brindando a fiabilidad que le ha caracterizado en términos generales durante sus casi tres siglos de existencia y le ha permitido sobrevivir» (Burgueño, 2010: 20-21).
\end{abstract}

Se deduce que el periodista necesita adaptarse constantemente a los cambios sociales y tecnológicos, aunque son los primeros los que deben marcar el día a día del profesional. Esa formación continua es clave para saberse adaptar a los tiempos, a los cambios, a los nuevos proyectos, a los diferentes soportes, por tanto, la profesión es ágil y dinámica.

Lo que se denominó en su momento nuevas tecnologías de la información y de la comunicación y que se ha transformado en un nuevo sistema de producción, distribución, consumo y retroalimentación de la información. Esas nuevas tecnologías se han consolidado instaurándose la cultura de la web 2.0 en la que cualquiera puede ser creador de contenidos, también informativos, utilizando unas herramientas sencillas desde el punto de vista del manejo tecnológico.

En esa línea se han planteado discusiones en diferentes foros sobre si se puede hacer periodismo sin periodistas y se ha acuñado el término, para nosotros un tanto desacertado, de periodismo ciudadano. Pese a todas esas novedades tecnológicas, la base es la misma: la objetividad, la imparcialidad, el uso correcto de las fuentes, contrastar lo que se publica... Es decir, la esencia de la profesión de periodista.

«Esta colaboración ciudadana en la difusión de noticias y de propuestas personales ha llevado a que en determinados sectores se hable de periodismo ciudadano, una expresión poco afortunada que no refleja la realidad. El periodismo exige niveles cultural y ético adecuados, capacidad de trabajo y de síntesis, lenguaje correcto, tenacidad, fuentes seguras y contrastadas, una firma fiable que garantice la veracidad y estructuras de trabajo profesionales. Pero también es cierto que en este contexto los periodistas tienen que asumir nuevos retos y elevar el nivel de su trabajo, además de coordinar el flujo informativo con exigencia de calidad, con los modelos textuales que brindan los géneros y con un rigor que ponga el sello de autenticidad» (Edo, 2009:15).

Las nuevas herramientas son solamente eso, herramientas, que deben ser comprendidas y utilizadas bajo criterios periodísticos.

«Pero difícilmente hay tecnología que pueda ofrecer alternativas que superen el dominio de las grandes ideas que durante el siglo XX ha tenido el periódico de calidad, con su tradición de investigación en profundidad de los hechos, con su capacidad de síntesis, con su defensa inspirada de las diversas cuestiones» (López: 2010: 50).

La tecnología ha invadido todos los ámbitos de la comunicación y ha cambiado sustancialmente el proceso informativo. Han aparecido nuevos medios, ha facilitado la creación de contenidos en este caso informativos sin que se tengan que ceñir a las estructuras rígidas de los medios de comunicación, con los blogs 
como herramienta inicial, y que han sido el germen de un intercambio informativo muy notable en las redes sociales y a través, por ejemplo, de Twitter. Pero, en definitiva, se trata de potenciar la interactividad y la convergencia mediática y tecnológica.

De esta forma, se supera con mucho la época analógica, la de los tradicionales medios de comunicación y, por tanto, también la formación tendrá que variar porque el perfil de profesional también ha cambiado. El mercado laboral es distinto, la producción de contenido es diferente, al igual que también la circulación de esa información.

De todas maneras, sería un error mayúsculo, como ha sucedido durante la última década, idolatrar a la tecnología. Nuestros alumnos deben ser capaces de adaptarse a las posibilidades que ésta le ofrece y sobre todo a las que les seguirá aportando en los próximos años con los vertiginosos cambios que se presuponen. Sin embargo, deben mantener las tradicionales habilidades que se le asignan a cualquier periodista de forma histórica y que deben tener carácter perdurable: selección, análisis, síntesis, verificación, contraste y jerarquización.

Muchos jóvenes profesionales están supliendo el enorme hueco dejado por las facultades en su formación con programas no reglados y relacionados con las nuevas técnicas de marketing o directamente con la tecnología, que sí están siendo desarrolladas y estudiadas de forma sistemática. El problema en esta ocasión es que, si las bases periodísticas no son lo suficientemente sólidas, en muchas ocasiones se confunden las tareas propias del periodismo con la propaganda o el marketing directo.

Por tanto, hay que asumir que no es suficiente con dominar estas nuevas tecnologías para trabajar en un medio digital o para ganarse la vida como periodista online, sino que logrará ser un buen profesional si sus informaciones disponen de calidad, rigor, contraste, credibilidad...

La situación de crisis económica que vive la Universidad condiciona también la sensación entre el estudiante de falta de incorporación de la última tecnología. Sin embargo, esta percepción debe ser superada y apostar por mostrar unas nuevas formas de hacer, pero sin olvidar ni pasar por alto los principios básicos del periodismo.

Lourdes Martínez (2005) apunta que en las redacciones de los cibermedios «predominan los periodistas jóvenes, con deficientes condiciones laborales, que realizan múltiples tareas, muchas de ellas bastante alejadas de la concepción del periodismo y más propias de otros profesionales como los diseñadores, los técnicos y los informáticos, trabajan toda su jornada delante del ordenador sin posibilidad prácticamente de hacer trabajo en la calle y, por lo general, tienen una deficiente preparación en periodismo digital».

Solo así, se logrará que se supere esa percepción de que la Universidad está anclada en el pasado en la formación de los próximos profesionales de la co- 
municación. Desde los centros educativos se ha de aceptar de forma definitiva que estamos en un cambio de paradigma de la comunicación que también nos afectará directamente a los estudios de Periodismo que no pueden quedarse anclados en el pasado como si los cambios no fueran con ellos.

La adaptación de las titulaciones al Espacio Europeo de Educación Superior no ha logrado, al menos a priori y en términos generales, romper con esa sensación de alejamiento del mundo profesional y del académico y para intentar resolver esta división tan clara que todavía hoy se percibe se debe realizar una profunda reflexión en el ámbito universitario que lleve a un cambio en el hacer para lograr una mejor formación de nuestros alumnos que les permite sobre todo tener una capacidad de adaptación a los cambios constantes que vive la profesión.

Pero incluso dentro de las propias propuestas de planes de estudios hay que realizar una reflexión crítica sobre la división existente y demasiadas veces inconexa entre las materias teóricas vinculadas a las ciencias de la comunicación y las de formación profesional, que son calificadas como prácticas o experimentales. Esa ruptura tradicional debería tender a desaparecer en los planes de estudios, aunque se constata como se mantiene cierta separación inexplicable en la formación de nuestros futuros comunicadores, muy críticos con esta situación.

El distanciamiento afecta también a los alumnos y se convierte en un déficit que se plantea cuando entran a formar parte del mundo laboral: la dificultad que tienen a la hora de tomar decisiones de forma razonada, justificada en ocasiones por falta de madurez y, en otras, por no recibir una formación encaminada a que puedan ser autónomos en la toma de decisiones, siempre de forma reflexiva. Este es uno de los objetivos que claramente deberían marcarse las facultades de Comunicación.

En esta línea, los conocimientos y los procedimientos básicos de la profesión nunca se deben modificar. Es decir, el tratamiento con las fuentes, la objetividad, la rigurosidad... ¿Cabría pensar en un médico que pensara que las nuevas tecnologías le eximen de curar a los enfermos?

José Manuel Burgueño (2010) señala que, al margen de las necesarias adaptaciones tecnológicas y de modelo de negocio, lo principal radica en mantener vivas las esencias del periodismo.

\footnotetext{
«Lo que le queda al periodismo son sus valores: la búsqueda de la verdad, la lealtad a los ciudadanos, la disciplina de la verificación, la independencia con respecto a aquellos de quienes informa, el control del poder, la oferta de un foro para la crítica y el comentario, la presentación sugerente y relevante, y la valoración proporcionada, el respeto a la conciencia de sus profesionales» (Burgueño, 2010: 237).
}

Por tanto, la base de la formación como profesional del periodismo no cambia, lo que varía son simplemente las herramientas que se utilizan. 
$\mathrm{Y}$ es en esas herramientas en las que hay que formar a los profesionales, ya sean nuevos graduados, licenciados o periodistas necesitados de un reciclaje, para que sepan, en todo momento, enfrentarse a los necesarios cambios.

No es algo novedoso, por otra parte. La aparición de nuevos soportes siempre ha provocado modificaciones en la profesión, pero no ha supuesto una revolución radical en la forma de hacer periodismo. Sólo en la forma de informar, de contar, de interpretar y explicar la realidad.

Son algunas de las cuestiones que hay que tener en cuenta. No se debe pasar por alto que esos cambios deben afectar (lo están haciendo ya, aunque a una escala muy baja todavía) también a las facultades de comunicación, que han de ser lo suficientemente flexibles como para saber convivir en ese mundo de continuos cambios. Ni la profesión ni la sociedad pueden admitir que buena parte de las facultades se mantengan todavía ancladas en modos y herramientas que son sinónimos del pasado.

Los planes de estudios vigentes en la actualidad no han conseguido adaptarse a la nueva realidad del periodismo, sino que ofrecen una visión de la práctica de la profesión que en la mayoría de las ocasiones está caduca y refleja modelos que ya no son aplicables en el día a día de los medios de comunicación y del resto de salidas profesionales para el periodista.

Este proceso de cambio vertiginoso, por medio de las nuevas tecnologías (que ya no son tan nuevas, sino que son las únicas que tienen y utilizan la inmensa mayoría de los periodistas y comunicadores para transmitir información), lo están viviendo los profesionales; lo están disfrutando y, en parte también, lo están sufriendo.

El periodista debe enfrentarse a su labor con un conocimiento suficiente de la tecnología. Como se acaba de señalar, es fundamental el uso de estas herramientas para desarrollar las viejas destrezas que se han asociado al periodismo (selección, análisis, síntesis, verificación, contraste y jerarquización). Además, para aquellos que trabajen en los medios digitales resulta necesario desarrollar otras como el dominio del lenguaje multimedia e hipertextual.

¿Es suficiente el dominio de las nuevas tecnologías para trabajar en un medio digital? La respuesta es tajante; de ninguna manera. En primer lugar, el periodista no debe olvidar nunca la veracidad y la calidad técnica de sus informaciones, como ya se ha expuesto. En segundo, limitar la formación al uso de una tecnología concreta es condenar a los alumnos a una obsolescencia casi programada, dado que su competencia terminaría con el fin de esas tecnologías. Hay que formar en conceptos y principios sólidos y permanentes; en capacidad de adaptación a las circunstancias tecnológicas y sociales.

Es más, como apunta López (2010), el fin último del periodismo no está solo en informar, formar y entretener, que son los que tradicionalmente se han apuntado. El periodismo es también un compromiso ético con la verdad, la libertad, 
el rigor, con el respeto a la integridad moral; y, en esta línea, debe fomentar sociedades más democráticas, plurales y participativas. Y ninguna de estas cuestiones se consigue únicamente gracias a la tecnología.

\section{Necesidad de emprender}

Uno de los principales aspectos que ha cambiado la crisis económica es la empresa periodística. Las grandes pasan por situaciones muy delicadas, gran parte de ellas con notables deudas generadas en su mayoría por inversiones no demasiado valoradas en tiempos de bonanza, y que están arrastrando a tradicionales medios de comunicación que, sin embargo, se podrían sustentar económicamente sin demasiados problemas. Son los casos, por ejemplo, de cabeceras como El País o El Mundo, que pese a que siguen siendo referentes informativamente están pasando por un momento delicado a causa de por las deudas de los grupos a los que pertenecen, además del descenso de la publicidad y de sus propias tarifas.

Difícilmente los jóvenes licenciados o graduados tendrán la puerta abierta en estos tradicionales medios de comunicación y deberán buscar una salida personal en otros mercados de la comunicación, que sí existen, pero que deberán explorarse todavía más. Es, por ejemplo, el de las pequeñas empresas de emprendeduría, prestadoras de servicios de comunicación a clientes cada día más diversos.

No es una tarea sencilla para los recién titulados enfrentarse a nuevos proyectos, pero sí es cierto que es una de las principales oportunidades que se están detectando en la actualidad. La dificultad es todavía mayor por la escasez de formación en la materia, aunque existan asignaturas en los planes de estudios vinculadas a proyectos y gestión de la empresa informativa.

Aspectos con la dificultad de gestión empresarial, la morosidad, la búsqueda de respuestas inmediatas, la falta de financiación externa y la escasa preparación en la materia, entre otros aspectos, complican y mucho que nuestros graduados o licenciados se animen a crear su propia empresa prestadora de servicios comunicativos.

En la parte positiva está que la inversión que se requiere es cada vez menor por el amplio catálogo de herramientas comunicativas existentes con la red como soporte, buena parte de ellas gratuitas o de muy bajo coste.

La idea de partida es que los tradicionales medios de comunicación ya no son los únicos intermediarios para transmitir información a la opinión pública. Este hecho abre multitud de oportunidades para este tipo de pequeñas empresas de comunicación. La creación de canales propios (blogs, Facebook, Twitter,...) son instrumentos extraordinarios para la transmisión de información, de opinión, de imagen..., es decir, herramientas de comunicación imprescindibles que deben ser gestionadas profesionalmente. 
De todas maneras, estos nichos de empleo no son excluyentes con la posibilidad de trabajar o colaborar con grandes medios de comunicación, sino que en el horizonte debe plantearse una combinación.

\begin{abstract}
«Acentuados los anteriores perfiles por la actual crisis, ha de verse en todo ello no sólo los riesgos y peligros, sino las ocasiones y oportunidades que se nos presentan para corregir errores y defectos, tanto en los procesos formativos como en la inserción laboral de los profesionales de la comunicación y, en definitiva, el funcionamiento mismo del sistema audiovisual. La superación de una falsa alternativa entre teoría y praxis, la capacidad de adaptación a los nuevos retos, la constante y dinámica mutación de conocimientos, el fomento de la creatividad, de la capacidad innovadora y el espíritu emprendedor, la creación de nuevas sinergias y alianzas son piezas claves del proceso» (Vivar y García, 2012).
\end{abstract}

En este proceso cambiante de incorporación al mercado laboral de nuestros licenciados/graduados, las propias facultades de comunicación deberían potenciar esta línea de trabajo. Como ejemplo, la Facultat de Ciències de la Comunicació de la Universitat Autònoma de Barcelona ha puesto marcha un proyecto piloto denominado TicLaude. Jóvenes Emprendedores Universitarios en TIC , que pretende ofrecer formación a los estudiantes de Periodismo, Comunicación Audiovisual y Publicidad y Relaciones Públicas que deseen desarrollar una idea innovadora en la materia y que posteriormente puedan presentar a un importante número de empresas e instituciones. Se enmarca dentro del proceso de formación continua que se considera deben tener los profesionales de la comunicación y sobre todo con la idea de facilitar la generación de autoempleo.

\title{
5. Propuestas de futuro
}

Las incertidumbres que se ciernen sobre el sector de los medios de comunicación hacen todavía más necesaria la colaboración estrecha entre Universidad y empresas, que en la actualidad se reduce a muy escasos foros de debate. Espacios, físicos o virtuales, en los que el nivel de diálogo sea absolutamente sincero.

La situación la analiza a la perfección Pablo Mancini en su acertadísimo libro Hackear el periodismo (2011). El periodista argentino describe una industria desorientada, asustada y atenazada, que no es capaz de reaccionar ante la gran cantidad de cambios que suceden a su alrededor. Mancini habla literalmente de estado de "perplejidad» en el que se encuentra el sector de la comunicación, desde las grandes empresas a la mayoría de los profesionales.

Esta situación fue descrita en abril de 2012 por Antonio Martín Hervás, consultor internacional de Innovation Consultin Group, en el cierre del I Seminario de Periodismo Visual de la Universidad San Jorge: «Las empresas están muy nerviosas

\footnotetext{
9 El Ministerio de Educación, a través de la Secretaría General de Universidades, concedió en diciembre de 2011 a la Facultat de Ciències de la Comunicació de la Universitat Autònoma de Barcelona la subvención para desarrollar el proyecto «Bolsa de jóvenes emprendedores universitarios europeos en TIC», en el marco de la Orden EDU/2346/2011, de 18 de agosto, de convocatoria de subvenciones para el desarrollo de proyectos y acciones orientadas a la mejora de la atención integral y empleabilidad de los estudiantes universitarios. Investigador principal: Josep Maria Català. Resto de investigadores: Santiago Tejedor, Virginia Luzón, Antoni Castel, Norminanda Montoya, Francesc Vilanova, Miguel Pérez Quintanilla y Fernando Sabés. Para más información se puede consultar http://www.ticlaude.info
} 
porque no saben qué hacer, no tienen ideas y las que tienen, no saben cómo aplicarlas» ${ }^{10}$.

«Es el miedo, es la incertidumbre que a algunos paraliza y a otros nos impulsa a experimentar y experimentar; incluso, ojala, más allá de lo razonable» (Mancini, 2011: 14), señala Gumersindo Lafuente, adjunto a la dirección de El País.

«El periodismo atraviesa un difícil proceso de reconversión, una crisis existencial con todas las de la ley. Mientras la industria se mueve como pollo sin cabeza en un entorno digital dominado por el todo gratis, los periódicos, en papel, buques insignia del sector, llevan camino de convertirse en piezas de museo, en una época especialmente dura con la prensa escrita» (Molina, 2011: 8)

Martín y Mancini coinciden en que «es más importante acercarse al oficio y probar, errar y lidiar con la experiencia, que dar por recibido a un futuro improbable que supuestamente llegó para quedarse» (Mancini, 2011: 101).

$\mathrm{Y}$ es precisamente en este sentido en el que las universidades pueden y deben convertirse en auténticos laboratorios periodísticos. Algunas experiencias existen ya, como el Laboratorio Multimedia de la Universidad de Navarra, la UAB Media de la Universidad Autónoma de Barcelona, o el proyecto de la Universidad San Jorge llamado Dragón Digital.

Algunos grandes medios han puesto en marcha contadas iniciativas de este tipo. The New York Times, Los Angeles Times, BBC o The Guardian son algunos de ellos, citados por Mancini (2011; 58-60). También los hay en pequeños medios, pero los ejemplos son todavía más escasos debido al desembolso económico que su supone. Precisamente son estos pequeños medios los que podrían desarrollar un trabajo compartido con las universidades, que redundaría en beneficio mutuo.

La profesión se ha dado cuenta también de ello, aunque todavía al margen de las grandes estructuras empresariales. Cabe destacar en este sentido dos iniciativas interesantes. La primera de ellas, el Congreso de Periodismo Digital que se celebra anualmente en Huesca desde el año 2001 y que es uno de los pocos foros en los que existe una coincidencia del mundo académico y el profesional para establecer diálogo, para compartir experiencias, retos e incertidumbres.

La otra experiencia es la que está desarrollando la Federación de Asociaciones de Periodistas de España (FAPE) a través de su revista Periodistas. La nueva orientación de esta publicación la ha convertido en un foro de debate y reflexión sobre cuestiones de interés para la profesión como la transparencia informativa de los gobiernos, la libertad de prensa, la situación laboral, los nuevos caminos de la industria comunicativa o la necesaria reorientación de la formación de los profesionales.

Experiencias que, en nuestra opinión, deberían amplificarse y en la que los medios de comunicación deberían estar presentes de manera decidida. Porque el

10 http://www.dragondigital.es/tag/antonio-martin-hervas/ 
futuro del periodismo o se inventa entre todos, o no se logrará. Resulta absurdo que cada medio esté tratando de encontrar la piedra filosofal por su cuenta, desaprovechando las potencialidades evidentes que tendría un trabajo colaborativo, con las facultades de comunicación como ejes articuladores del mismo y con las asociaciones de periodistas como elementos fundamentales y garantes del mantenimiento de los principios que han hecho posible el sostenimiento de esta profesión a lo largo de los últimos siglos.

En este sentido, una propuesta concreta final sería la creación de clústers periodísticos ligados a territorios o ámbitos concretos, en los que todos los actores pudieran sumar fuerzas. En nuestra opinión, esta fórmula, que ya se ha desarrollado de manera exitosa en sectores muy diversos, aportaría valores indudables al sector, tanto desde el punto de vista de la industria como del factor humano.

En concreto, consideramos que permitiría concretar y determinar con mayor facilidad los retos estratégicos del sector, marcar objetivos más ambiciosos que las actuales empresas (asustadas y debilitadas) no son capaces de fijar; permitiría percibir oportunidades y sinergias entre profesionales y empresas, fijar los elementos que aportan valor real a la información dentro de la cadena o incrementar la confianza interna del sector y ser capaces, en conjunto, de resistir mejor las crisis. Una cuestión del máximo interés sería recuperar la confianza de la sociedad hacia el periodismo, por medio de un proceso que permita al sector conocer y comprender mejor su entorno, al mismo tiempo que debería reducir los elementos que en las últimas décadas han ido minando el crédito de la sociedad hacia el periodismo. Por último, la formación de un clúster permitiría contar con un elemento de interlocución interno, pero también con la Administración y con otros sectores; algo que en la actualidad no son capaces de hacer por sí mismas ni las asociaciones de periodistas, ni los sindicatos ni las diferentes organizaciones patronales.

\section{Bibliografía}

Amaral, F. y Gimeno, D. (2009): Evolución, tendencias y modelos en el diseño de webs de noticias. Barcelona: Sol90.

Burgueño, José Manuel (2010). Cuestión de confianza. La credibilidad, el último reducto del periodismo del siglo XXI. Barcelona: Editorial UOC.

Casals Carro, María Jesús (2006). La enseñanza del periodismo y las nuevas tecnologías de la información y la comunicación. Estudios sobre el Mensaje Periodístico, núm. 12. Madrid: Universidad Complutense de Madrid, 59-70.

Díaz Noci, Javier (2008). Ciberperiodismo, investigación y docencia: una oportunidad única. En Anàlisi, núm. 36. Barcelona: Servicio de Publicaciones.

Edo, C. (2009). La noticia en Internet: cibermedios, blogs y entornos comunicativos emergentes. En: Estudios de Periodística XV. Madrid: SEP. 
Estudio sobre inversión publicitaria en medios digitales. Resultados del año 2011. Interactive Advertising Bureau (IAB Spain), Marzo de 2012. Consulta: http://www.iabspain.net/wp-content/uploads/downloads/2012/05/Estudio_inversion_publicidad_IAB_Spain_2011.pdf

Flores J. y Aguado, G. (2005). Modelos de negocio en el ciberperiodismo. Madrid: Fragua.

Libro Blanco de los títulos de grado en Ciencias de la Comunicación. Consultado en: http://www.aneca.es/activin/docs/libroblanco_comunicacion_def.pdf

López García, Xosé (2011). Las mudanzas periodísticas. En Telos, Cuadernos de comunicación e innovación, núm. 86, enero-marzo. http://sociedadinformacion.fundacion.telefonica.com/seccion=1266\&idioma=es _ ES\&id=2011012511370001 \& activo $=6$.do

López García, Xosé (2010). La metamorfosis del periodismo. Historia de los que permanece y lo que cambia en el ciberperiodismo del tercer milenio. Sevilla / Zamora: Comunicación Social.

Mancini, Pablo (2011). Hackear el periodismo. Manual de laboratorio. Buenos Aires: La Crujía Ediciones.

Martínez Albertos, José Luis (1997): El ocaso del periodismo. Barcelona: CIMS, Martínez Rodríguez, L. (2005). El periodista en transformación en el ciberespacio: la reivindicación del periodista como periodificador en la red. En: Razón y Palabra. Consultado en: www.cem.itesm.mx

Micó, Josep Lluís (2006). Periodisme a la Xarxa. Llenguatgenoud’unoficivell. Vic: Eumo Editorial.

Molina, Marta (2011). ¡Que paren las rotativas! En: Periodistas, núm 24. Madrid: FAPE.

Pastor, María Ángeles (2010). Quiero ser periodista: tras las motivaciones de la profesión periodística. Comunicar. En Comunicar, Revista Científica de Ecocomunicación. Huelva: Grupo Comunicar, 191-200.

Peinado Miguel, Fernando y Fernández Sande, Manuel (2011). Reflexión sobre la motivación de los alumnos de Grado en la elección de estudios de comunicación en las universidades de Madrid. En: Estudios sobre el mensaje periodístico, Vol. 17, núm. 2. Madrid: Servicio de Publicaciones de la Universidad Complutense, 383-400.

Pestano Rodríguez, José Manuel; Rodríguez Wangüemert, Carmen y Del Ponto, Patricia (2011). Transformaciones en los modelos de formación de periodistas en España. El reto europeo. En: Estudios sobre el mensaje periodístico, Vol. 17, núm. 2. Madrid: Servicio de Publicaciones de la Universidad Complutense, 401-415. 
Sabés, F.; Verón, J.J. (2008). Las nuevas tecnologías en la formación del periodista: una necesidad real e inmediata. En: Anàlisi. Bellaterra: Servei de Publicacions de la UAB, 105-114.

Salaverría, R. (2005). Cibermedios. El impacto de Internet en los medios de comunicación en España. Sevilla: Comunicación Social Ediciones y Publicaciones.

Salaverría, R. y Negredo, S. (2008). Periodismo integrado. Convergencia de medios y reorganización de redacciones. Barcelona, Editorial Sol 90.

Smith, Anthony (2011). ¿Es este el futuro del periódico? En Telos. Cuadernos de comunicación e innovación, núm. 86. Madrid: Fundación Telefónica.

Tejedor Calvo, Santiago (2006). Ciberperiodismo y Universidad: la inclusión curricular del periodismo on-line. En: Análisis y propuesta en torno al periodismo digital. VII Congreso Nacional de Periodismo Digital. Huesca: Congreso Nacional de Periodismo Digital.

Valera, S. (2008). Del becario precario a la redacción precaria. En: Periodistas. Madrid: FAPE, 6-9.

Valera, S. (2008). La crisis también se ceba con los periodistas. En: Periodistas. Madrid: FAPE, 6-9.

Vivar-Zurita, H. y García-García, A. (2012): La adaptación de la formación reglada y continua a la Comunicación. En: Revista Latina de Comunicación Social, 67. Consultado en: http://www.revistalatinacs.org/067/art/959_Madrid/15_Hipolito.html

\section{Referencia de este artículo}

Sabés Turmo, Fernando y Verón Lassa, José Juan (2012). Universidad y empresa ante la doble crisis del periodismo tradicional. Propuestas y reflexiones sobre la modificación sustancial del escenario periodístico. El clúster periodístico. En: adComunica. Revista Científica de Estrategias, Tendencias e Innovación en Comunicación, $\mathrm{n}^{\mathrm{0}} 4$. Castellón: Asociación para el Desarrollo de la Comunicación adComunica, Universidad Complutense de Madrid y Universitat Jaume I, 151170. DOI: http://dx.doi.org/10.6035/2174-0992.2012.4.10 